\title{
mTORC1 activation triggers the unfolded protein response in podocytes and leads to nephrotic syndrome
}

\author{
Noriko Ito ${ }^{1}$, Yukino Nishibori ${ }^{1}$, Yugo Ito ${ }^{1}$, Hisashi Takagi ${ }^{1}$, Yoshihiro Akimoto ${ }^{2}$, Akihiko Kudo ${ }^{2}$, Katsuhiko Asanuma ${ }^{3}$, \\ Yoshimichi Sai ${ }^{4}$, Ken-ichi Miyamoto ${ }^{4}$, Hitoshi Takenaka ${ }^{5}$ and Kunimasa Yan ${ }^{1}$
}

Although podocyte damage is known to be responsible for the development of minimal-change disease (MCD), the underlying mechanism remains to be elucidated. Previously, using a rat MCD model, we showed that endoplasmic reticulum (ER) stress in the podocytes was associated with the heavy proteinuric state and another group reported that a mammalian target of rapamycin complex 1 (mTORC1) inhibitor protected against proteinuria. In this study, which utilized a rat MCD model, a combination of immunohistochemistry, dual immunofluorescence and confocal microscopy, western blot analysis, and quantitative real-time RT-PCR revealed co-activation of the unfolded protein response (UPR), which was induced by ER stress, and mTORC1 in glomerular podocytes before the onset of proteinuria and downregulation of nephrin at the post-translational level at the onset of proteinuria. Podocyte culture experiments revealed that mTORC1 activation preceded the UPR that was associated with a marked decrease in the energy charge. The mTORC1 inhibitor everolimus completely inhibited proteinuria through a reduction in both mTORC1 and UPR activity and preserved nephrin expression in the glomerular podocytes. In conclusion, mTORC1 activation may perturb the regulatory system of energy metabolism primarily by promoting energy consumption and inducing the UPR, which underlie proteinuria in MCD.

Laboratory Investigation (2011) 91, 1584-1595; doi:10.1038/labinvest.2011.135; published online 29 August 2011

KEYWORDS: energy; mTOR; podocyte; signaling; UPR

The homeostasis of protein expression in cells is fundamentally required for physiological functions and is highly regulated by a dynamic equilibrium between the synthesis and degradation systems. ${ }^{1,2}$ After their synthesis in the endoplasmic reticulum (ER)-associated ribosomes, only accurately folded proteins are allowed to exit the ER and are transported to the Golgi compartment, whereas misfolded proteins are either retained in the ER in complex with the molecular chaperones or are degraded in a process referred to as ER-associated degradation. ${ }^{3}$ Under certain pathological conditions, the accumulation of unfolded proteins exceeds the folding/processing capacity of the ER. This ER imbalance, ER stress, triggers the specific signaling pathway, the unfolded protein response (UPR), which promotes cell survival by arresting protein translation and stimulating the expression of molecular chaperones. ${ }^{4}$

It has become evident that ER stress underlies the pathogenesis of a number of diseases; ${ }^{5,6}$ however, its role in the development of kidney diseases has been recognized only relatively recently. ${ }^{7,8}$ In a previous study, we implicated ER stress in the development of the rodent model of minimalchange disease (MCD). ${ }^{9}$ There are a number of studies suggesting that podocyte damage underlies the development of MCD; $;^{10,11}$ however, the pathophysiological mechanisms of proteinuria have not been fully elucidated. In the rodent puromycin aminonucleoside (PAN) model of MCD, we showed upregulation of GRP78, a molecular chaperone stimulated by ER stress, in podocytes on days 4 and 5 at a heavy proteinuric state; we also showed that this GRP78 upregulation was associated with a change in the cellular localization of nephrin from the plasma membrane to the cytoplasm. ${ }^{9}$ Nephrin is a transmembrane glycoprotein that is produced by podocytes and is responsible for the permselective barrier of the glomerulus. ${ }^{12,13}$ We previously determined that nephrin trafficking to the plasma membrane required $\mathrm{N}$-glycosylation in the $\mathrm{ER}^{14}$ and showed that a lack

\footnotetext{
${ }^{1}$ Department of Pediatrics, Kyorin University School of Medicine, Mitaka, Tokyo, Japan; ${ }^{2}$ Department of Anatomy, Kyorin University School of Medicine, Mitaka, Tokyo, Japan; ${ }^{3}$ Department of Internal Medicine, Juntendo University School of Medicine, Hongo, Tokyo, Japan; ${ }^{4}$ Department of Hospital Pharmacy, Kanazawa University, Kanazawa, Japan and ${ }^{5}$ Department of Biochemistry, Kyorin University School of Medicine, Mitaka, Tokyo, Japan Correspondence: Professor K Yan, MD, PhD, Department of Pediatrics, Kyorin University School of Medicine, 6-20-2 Shinkawa, Mitaka, Tokyo 181-8611, Japan. E-mail: kuniyan@ks.kyorin-u.ac.jp 
of ATP induced the hypoglycosylation of nephrin and its retention in the ER, which coincided with a strong induction of GRP78. ${ }^{15}$ Interestingly, glucocorticoids and the immunosuppressant mizoribine, which are commonly used to treat MCD, normalized the abnormal $N$-glycosylation and nephrin localization by restoring ATP levels. ${ }^{9,15}$ Thus, the balance of the energy regulatory system must have a crucial role in the correct processing of podocyte-producing proteins, and the imbalance of the energy regulatory system may induce ER stress and the UPR, which possibly underlies proteinuria in MCD. Then a question arose as to what events are the triggers to cause the UPR. An interesting previous study showed that pre-treatment with a mammalian target of rapamycin complex 1 (mTORC1) inhibitor protected against proteinuria in PAN-induced MCD, although the investigators proposed that its protective effect was due to a reduction in macrophage invasion. ${ }^{16}$ The mTORC1 signaling is a central pathway of protein synthesis system that consumes intracellular energy. ${ }^{17-20}$ Here, we show that the UPR in podocytes is a cause, not a concurrent event, of proteinuria in rat MCD model. We further show that activation mTORC1 acts as the upstream of the UPR by driving energy consumption in rat MCD and cultured podocytes.

\section{MATERIALS AND METHODS Antibodies and Reagents}

The anti-nephrin rabbit polyclonal antibody (pAb2) was used as previously described. ${ }^{9,14,15}$ The following antibodies were purchased from the suppliers as indicated: anti-phospho-S6 ribosomal protein (S6RP) (Ser235/236) rabbit monoclonal, anti-S6RP rabbit monoclonal (5G10), anti-phospho-p70S6K (Thr389) rabbit polyclonal, anti-p70S6K rabbit polyclonal, anti-phospho-4EBP1 (ser65) rabbit polyclonal, anti-4EBP1 rabbit polyclonal, anti-phospho-AMP-activated protein kinase $\alpha(\mathrm{AMPK} \alpha)(\mathrm{Thr} 172)$ rabbit monoclonal, anti-AMP$\mathrm{K} \alpha$ rabbit polyclonal, anti-phospho- $\alpha$-subunit of eukaryotic translation initiation factor $2 \alpha$ (eIF2 $\alpha)$ (Ser51) rabbit monoclonal and anti-eIF2 $\alpha$ mouse monoclonal (L57A5) (Cell Signaling Technology, Beverly, MA, USA); anti-phospho-AKT1 (Ser473) rabbit polyclonal (Rockland, Gilbertsville, PA, USA); anti-glucose-regulated protein 78 mouse monoclonal (Stressgen Biotechnologies, Victoria, Canada); anti- $\beta$-actin mouse monoclonal (Sigma, Tokyo, Japan); HRPlabeled goat anti-rabbit and anti-mouse immunoglobulins (Dako, Carpinteria, CA, USA); and Alexa Fluor 488-conjugated goat anti-rabbit IgG, Texas Red-X goat anti-mouse IgG (Molecular Probes, Eugene, OR, USA). PAN was purchased from Sigma. Microemulsion formulations (selfmicroemulsifying drug delivery system) of the mTOR inhibitor, everolimus and the vehicle were kindly donated by Novartis Pharma AG (Basel, Switzerland). Ascomycin and rapamycin were purchased from Wako Pure Chemical Industries (Osaka, Japan) and Sigma-Aldrich (St Louis, MO, USA), respectively. HPLC grade methanol was purchased from Nacalai Tesque
(Kyoto, Japan). Ammonium acetate was purchased from Wako Pure Chemical Industries.

\section{PAN Nephrosis}

The experimental protocol was approved by the Animal Care Committee of Kyorin University School of Medicine. Male Sprague-Dawley rats that weighed 150-200 g were purchased from Saitama Experimental Animal Supply (Sugito, Saitama, Japan). The number of the rats examined in this study was listed in the Supplementary Table. PAN nephrosis was induced by a single intravenous injection of PAN $(120 \mathrm{mg} / \mathrm{kg}$ body weight), and the rats were euthanized on day 5 . The administration of everolimus and vehicle occurred once daily via gavage at a dose of $4 \mathrm{mg} / \mathrm{kg}$ body weight from 1 day before the PAN injection until day 5 .

For the quantification of urinary albumin, urine samples were separated by 10\% SDS-PAGE, followed by Coomassie brilliant blue staining. ${ }^{21}$ The band that corresponded to a density of approximately $60 \mathrm{kDa}$ (albumin) was measured using NIH Image ver.1.63, and the urinary albumin to urinary creatinine excretion ratio was calculated based on measurements from the Jaffe assay.

\section{Cell Culture}

A conditionally immortalized mouse podocyte cell line was propagated and maintained as previously described. ${ }^{22}$ Cells were cultured in the absence or presence of PAN $(5 \mu \mathrm{g} / \mathrm{ml})$ in a time-dependent manner in RPMI-1640 medium that contained $100 \mathrm{U} / \mathrm{ml}$ penicillin/streptomycin supplemented with $5 \%$ fetal bovine serum at $37^{\circ} \mathrm{C}$. For mTORC1 inhibition experiment, cells were cultured in the presence of everolimus at the concentration of $5 \mathrm{ng} / \mathrm{ml}$ for $30 \mathrm{~min}$ before PAN incubation. Cells were lysed on ice in lysis buffer, ${ }^{22}$ and then protein samples were subjected to western blot analysis as described below.

\section{Immunohistochemistry}

Kidney cortex samples from rats were fixed in $4 \%$ paraformaldehyde for $2 \mathrm{~h}$ at $4{ }^{\circ} \mathrm{C}$ and embedded in paraffin wax. The slides were dewaxed, washed with Tris-buffered saline (TBS), and subjected to autoclave heating at $120^{\circ} \mathrm{C}$ for $10 \mathrm{~min}$ in Target Retrieval Solution (Dako) for antigen retrieval. Slides were incubated with $1 \% \mathrm{H}_{2} \mathrm{O}_{2} / \mathrm{TBS}$ for $30 \mathrm{~min}$ and then treated with blocking buffer (3\% BSA and $0.05 \%$ Tween-20 in TBS) for $60 \mathrm{~min}$. The slides were then incubated with anti-GRP78 $(2 \mu \mathrm{g} / \mathrm{ml})$ at $4{ }^{\circ} \mathrm{C}$ overnight. The immune complexes were detected using an avidin-biotin complex detection system (ABC KIT, Vector Laboratories, Burlingame, CA, USA).

\section{Dual Immunofluorescence and Confocal Microscopy}

For the dual immunostaining of GRP78 and pS6RP or nephrin, the slides were processed as described above. The reactions of the primary antibodies, GRP78 $(2 \mu \mathrm{g} / \mathrm{ml})$ and pS6RP $(1: 100)$ or polyclonal nephrin antibody $(5 \mu \mathrm{g} / \mathrm{ml})$ 
occurred overnight at $4{ }^{\circ} \mathrm{C}$, and the immunocomplexes were visualized using Alexa Fluor 488-conjugated goat anti-rabbit IgG and Texas Red-X goat anti-mouse IgG, respectively. Slides were examined under a confocal laser scanning microscope that was equipped with a Krypton/Argon laser (MRC1024; Bio-Rad, Hercules, CA, USA).

\section{Western Blot Analysis}

Rat glomeruli were isolated using the magnet beads perfusion method as described previously. ${ }^{23}$ Isolated glomeruli were homogenized and lysed on ice in lysis buffer as described above. Protein samples from the glomeruli and cultured podocytes were separated on $7.5 \%$ and $4-20 \%$ gradient gels under reducing conditions. Membranes were reacted with the primary antibodies for $4{ }^{\circ} \mathrm{C}$ overnight. After washing with $0.05 \%$ Tween-20 in TBS, membranes were incubated with HRP-labeled goat anti-rabbit antibody or anti-mouse antibody and the labeling was detected using the Western Lightning Chemiluminescence reagent kit (Life Science Products, Boston, MA, USA). All images were analyzed using the software using NIH Image ver.1.63.

\section{mRNA Expression Determined Using Quantitative Real-Time Reverse Transcriptase-PCR}

The messenger RNA was extracted from isolated glomeruli, and the expression levels of NPHS1 and GAPDH, the housekeeping gene that was used here for normalization, were verified using the TaqMan gene expression system (Applied Biosystems, Foster City, CA, USA) and a 7500 RealTime Polymerase Chain Reaction System (Applied Biosystems) according to the manufacturer's instructions. Each sample $(n=3)$ was run in triplicate.

\section{Measurement of Adenine Nucleotides}

The levels of the adenine nucleotides were determined using high-performance liquid chromatography with the Supelcosil LC-18-T HPLC column (Spelco, Bellefonte, CA, USA) set for the Shimadzu HPLC system (CBM-10A/LC-10AD, Shimadzu, Kyoto, Japan), which is equipped with a diode array spectrophotometer (SPD-M10AVP). Separation was conducted with a linear gradient of $0.1 \mathrm{M} \mathrm{KH}_{2} \mathrm{PO}_{4}+4 \mathrm{mM}$ tetrabutylammonium hydrogen sulfate ( $\mathrm{pH}$ 6.0) (solution $\mathrm{A}$ ) and a 7:3 mixture of solution A: methanol as described in the manufacturer's instructions (Spelco bulletin 913). The adenine nucleotides were determined based on their retention time and their peak wavelength. A molar extinction coefficient of 15400 at $259 \pm 0.5 \mathrm{~nm}$ was employed to estimate the concentration of the adenine nucleotides.

\section{Measurement of Everolimus Concentration}

Levels of everolimus in the serum samples were measured using the liquid chromatography/mass spectrometry (LCMS) system (LCMS-2020, Shimadzu) as recently described ${ }^{23}$ but with minor modifications. Briefly, serum samples from the rats treated with everolimus or vehicle on day 5 were taken $2 \mathrm{~h}$ after gavage. Aliquots of sera were mixed with 2.5 vol. of protein precipitation reagent and deproteinized supernatants (10 il) were analyzed. The mobile phase was delivered at $0.3 \mathrm{ml} / \mathrm{min}$. Single-ion-monitoring mode was used for analyte quantitation by simultaneously monitoring $\mathrm{Na}^{+}$-adducts; m/z 981 for [everolimus-Na] ${ }^{+}, \mathrm{m} / \mathrm{z} 937$ for [rapamycin-Na] $^{+}$and $\mathrm{m} / \mathrm{z} 815$ for [ascomycin-Na] $^{+}$.

\section{Statistical Analysis}

Data were provided as means \pm s.e.m. The statistical significance of the differences was calculated using the twotailed Mann-Whitney $U$-test. Only results with $P<0.05$ were considered statistically significant.

\section{RESULTS}

\section{ER Stress Precedes Proteinuria in PAN Nephrosis}

Determining whether ER stress causes proteinuria or is simply associated with it is important for understanding the pathogenesis of proteinuria in MCD. As our previous study examined the upregulation of GRP78 in the heavy proteinuric state of PAN nephrosis only, ${ }^{9}$ we reexamined the precise time course of ER stress through the progress of proteinuria. A significant proteinuria was initiated on day 2 after PAN injection (Figure 1). The majority of the GRP78 staining in the control glomeruli was observed in the cytoplasm of the podocyte cell bodies, whereas its staining area extended into the podocyte cell processes in the glomeruli on day 1 (Figure 2a). On and after day 2, the GRP78 expression tended to further increase (Figure 2a). On the other hand, nephrin expression in the control glomeruli was revealed to be exclusive glomerular basement membrane (GBM)-pattern (Figure $2 \mathrm{~b}$ ). In contrast, the overall distribution of nephrin in days 1 and 2 glomeruli consisted of GBM-pattern and podocyte cytoplasmic-pattern, where clear overlap of cytoplasmic nephrin with GRP78 was determined by dual immunofluorescence and confocal microscopy (Figure 2b, arrow). On days 3 and 5, GBM-pattern nephrin was drastically reduced and the majority of nephrin was revealed to be cytoplasmic-pattern, which was colocalized with GRP78 (Figure 2b, arrow). To semi-quantitatively compare
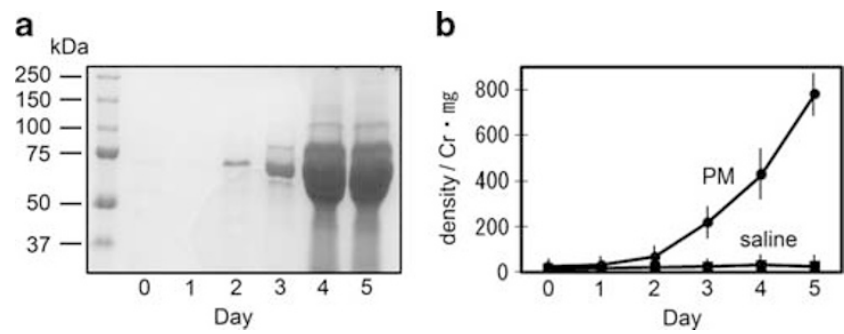

Figure 1 Urinary protein in PAN nephrosis. (a) Electrophoresis followed by Coomassie blue staining. A $10 \mu \mathrm{l}$ aliquot of each urine sample was subjected to SDS-PAGE. Obvious albuminuria was apparent from day 2. (b) Semi-quantitative analysis of the urinary protein revealed by the density of Coomassie blue staining/ $\mathrm{Cr}(\mathrm{mg})$ ratio. 
a
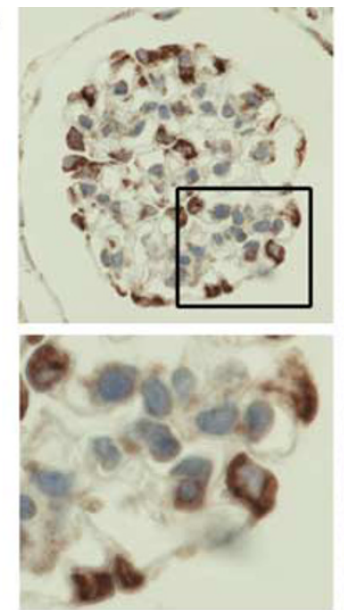

control
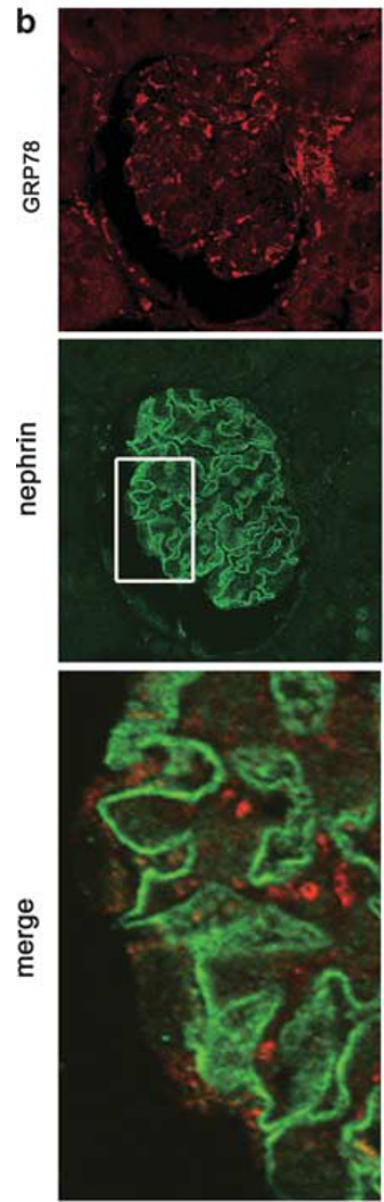

control
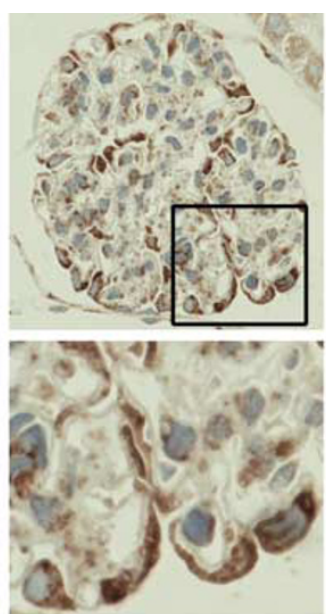

day 1
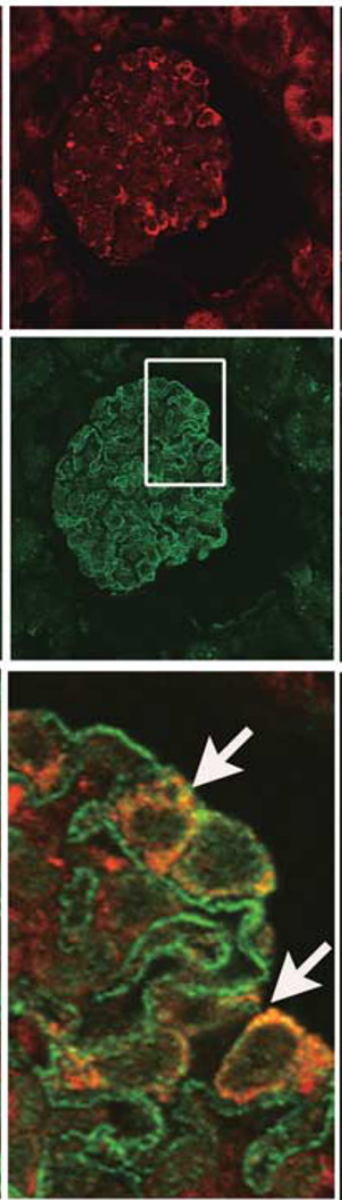

day 1
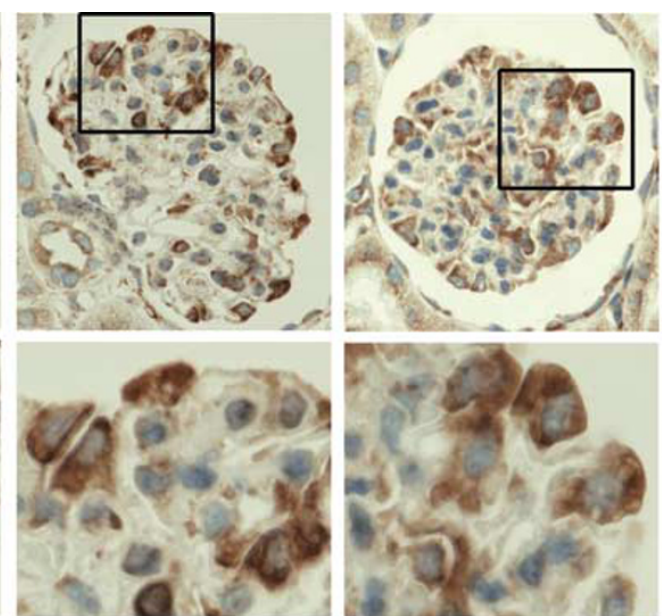

day 2

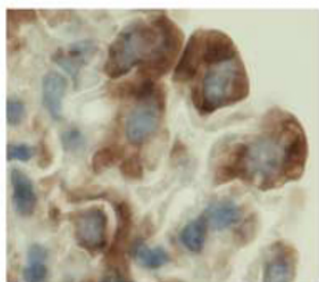

day 3
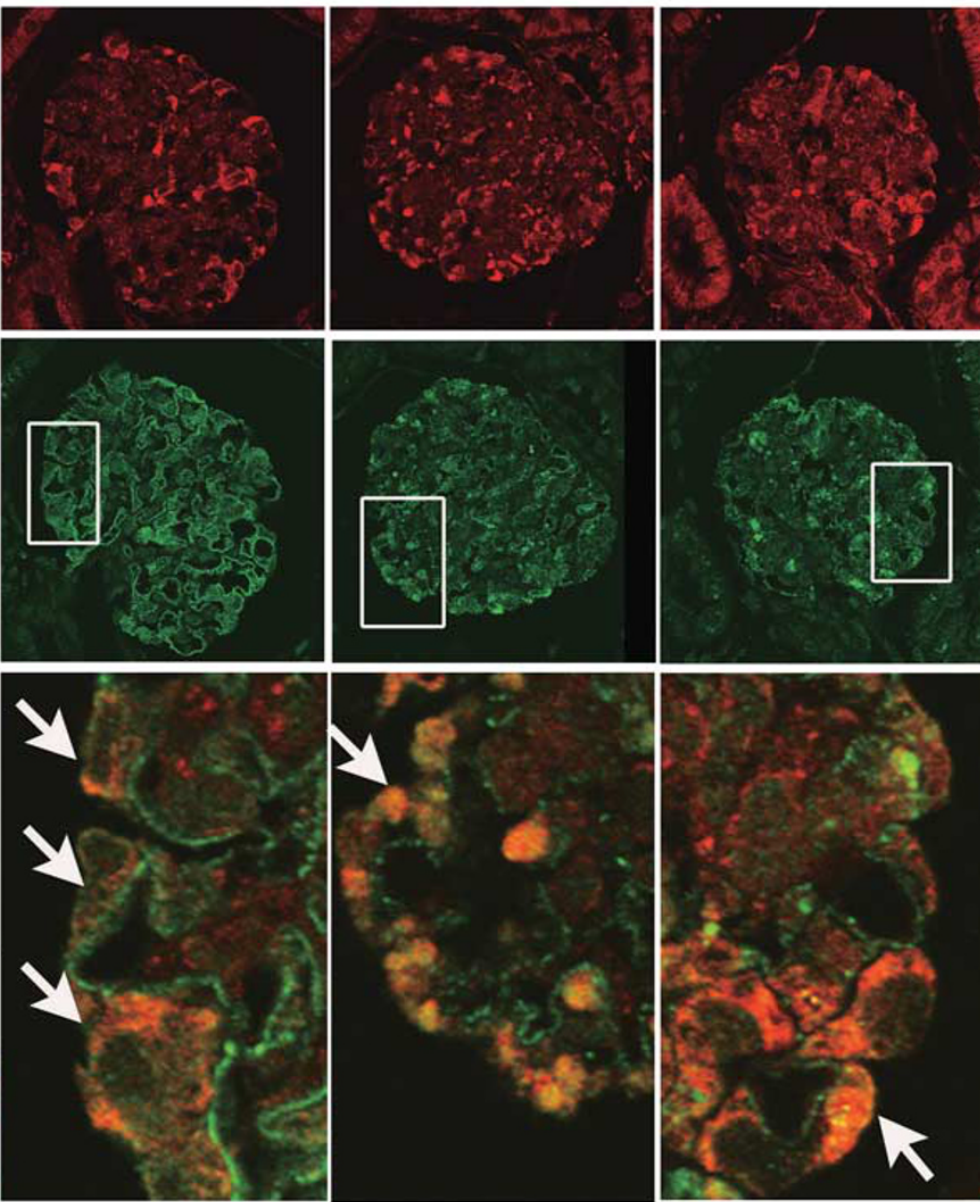

day 2

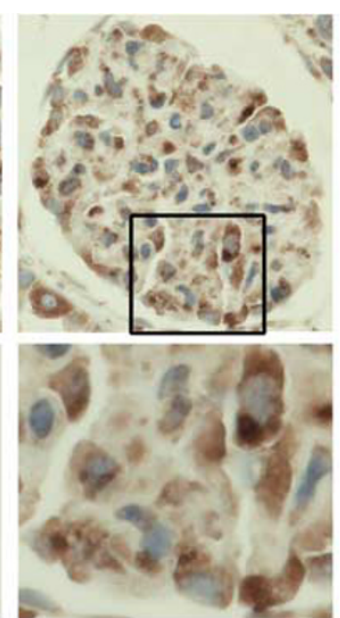

day 5
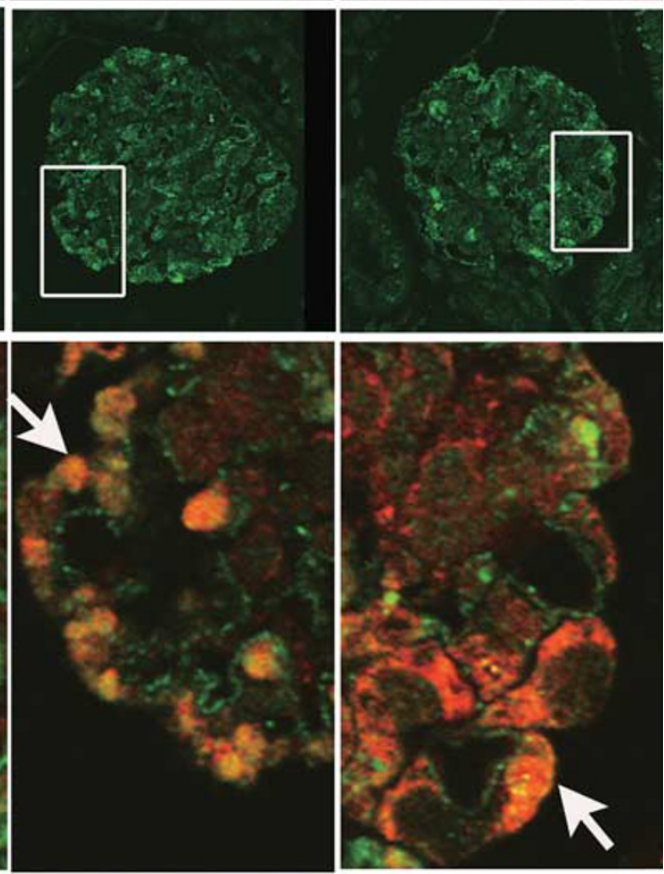

day 3

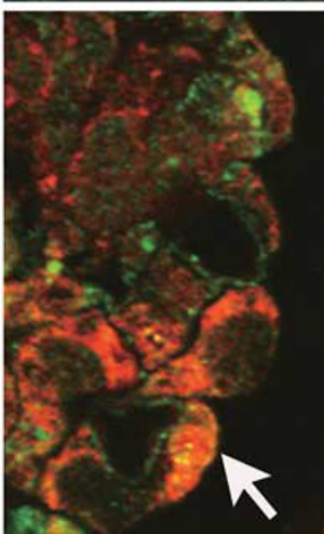

day 5

Figure 2 The time course of GRP78 and nephrin expression during PAN nephrosis. (a) GRP78 immunohistochemistry in the kidney sections from PAN nephrosis samples. Each rectangle of the upper panel was enlarged in the under panel. (b) Confocal microscopy images of the PAN glomeruli dualimmunostained with anti-nephrin antibody (green) and anti-GRP78 antibody (red). Each rectangle area of the middle panel was merged with the same part of the upper panel, and enlarged in the under panel. Orange color indicates colocalization of nephrin (green) with GRP78 (red). Original magnification, $\times 400$ (a, b).

the nephrin expression at a protein level, we next examined western blot using the glomerular samples. As shown in Figure 3a, nephrin of the control glomeruli consisted of double bands, corresponding to $\sim 200 \mathrm{kDa}$ and $\sim 175 \mathrm{kDa}$, which are speculated to be a plasma membrane form and a ER form, respectively. ${ }^{9,14,15}$ The upper band nephrin 

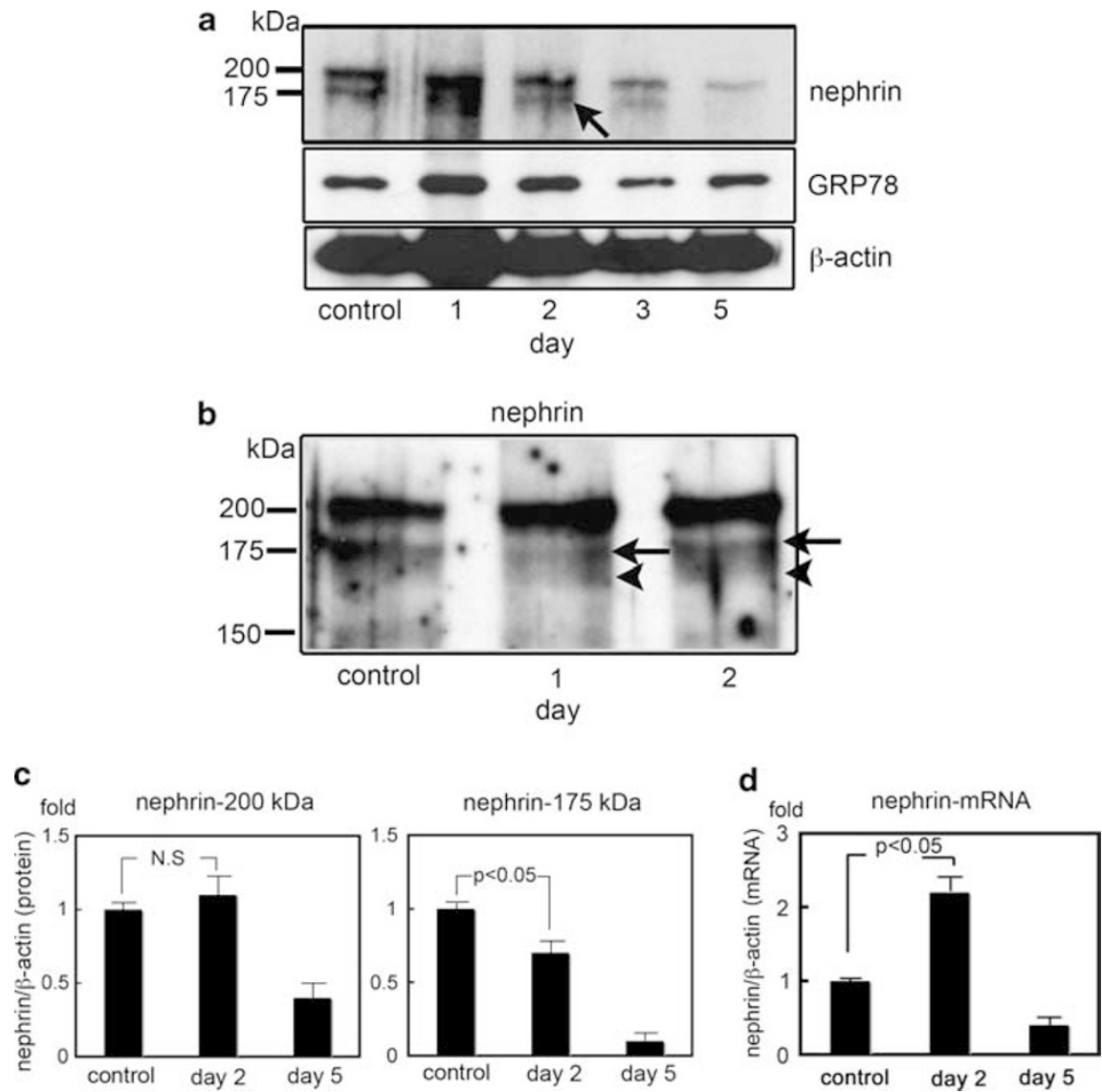

Figure 3 Protein and mRNA expression of nephrin and GRP78 in the glomeruli from control and PAN nephrosis rats. (a) Western blot analysis. In all, $50 \mu \mathrm{g}$ protein of isolated glomerular samples from PAN nephrosis and control rats was analyzed. $\beta$-Actin was applied as the internal control. Apparent reduction of $\sim 175-\mathrm{kDa}$ nephrin in the day 2 glomeruli was seen (arrow). (b) In all, $100 \mu \mathrm{g}$ protein of other samples besides (a) were subjected to SDS-PAGE and blotted for nephrin. There are clear ladder-like migrations that is faster than $\sim 175$-kDa nephrin in days 1 and 2 glomeruli. (c) Semiquantitative analysis for the protein expression of $\sim 200-\mathrm{kDa}$ nephrin and $\sim 175-\mathrm{kDa}$ nephrin. Comparison was only performed between control and day 2 . Mean \pm s.e.m., $n=3$ rats. (d) Real-time RT-PCR. The same glomerular samples ( $n=3$ per group) from (c) were applied, and the expression ratio was normalized to GAPDH. The NPHS1/GAPDH ratio of the control glomeruli was assigned a value of 1.0 .

( 200-kDa nephrin) was certain among the control and days 1 and 2 glomeruli; however, its expression was apparently reduced from day 3 glomeruli (Figure $3 a$ ). On the other hand, it was of great interest that the intensity of $\sim 175-\mathrm{kDa}$ nephrin of day 2 and day 3 glomeruli was distinctly reduced compared with the control glomeruli (Figures $3 \mathrm{a}$ and b, arrow). Moreover, the ladder-like migration that was faster than $175-\mathrm{kDa}$ nephrin was also identified in the samples from day 1 and day 2 glomeruli (Figure 3b, arrowhead). When compared with the control glomeruli, although semi-quantitative analysis found no significant difference of $\sim 200-\mathrm{kDa}$ nephrin in day 1 glomeruli (data not shown) and day 2 glomeruli, there was a significant reduction of $\sim 175-\mathrm{kDa}$ nephrin in day 1 (data not shown) and day 2 glomeruli (Figure 3c). Quantitative RT-PCR using the same glomerular samples detected a significant upregulation of nephrin mRNA in day 2 glomeruli compared with the control glomeruli (Figure 3d), which indicates that a reduction in
175-kDa nephrin protein expression on day 2 occurred via regulation at the post-translational level.

\section{mTORC1 is Associated with ER Stress in PAN Nephrosis}

Given the new finding that ER stress preceded a significant reduction of $200-\mathrm{kDa}$ nephrin that coincided with heavy proteinuria, we further investigated the triggers that could evoke ER stress. The mTORC1 signaling pathway stimulates cellular growth and proliferation through energy-consuming processes, and this signaling pathway stimulation is achieved via the phosphorylation of the downstream effectors p70S6K, 4E-BP1 and S6-RP. ${ }^{17-20}$ Thus, we hypothesized that this energy-consuming process, which leads to a lack of ATP in the podocytes, results in UPR activation, which is an energy-saving process. Immunohistochemistry using the phosphorylated form of S6RP (p-S6RP) revealed its low expression in some of the podocytes in control glomeruli (Figure 4). On day 1, p-S6RP expression was drastically 


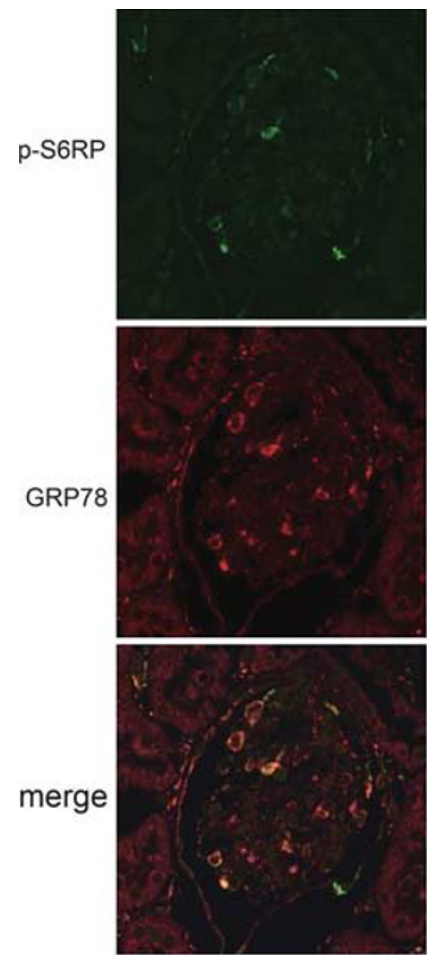

control
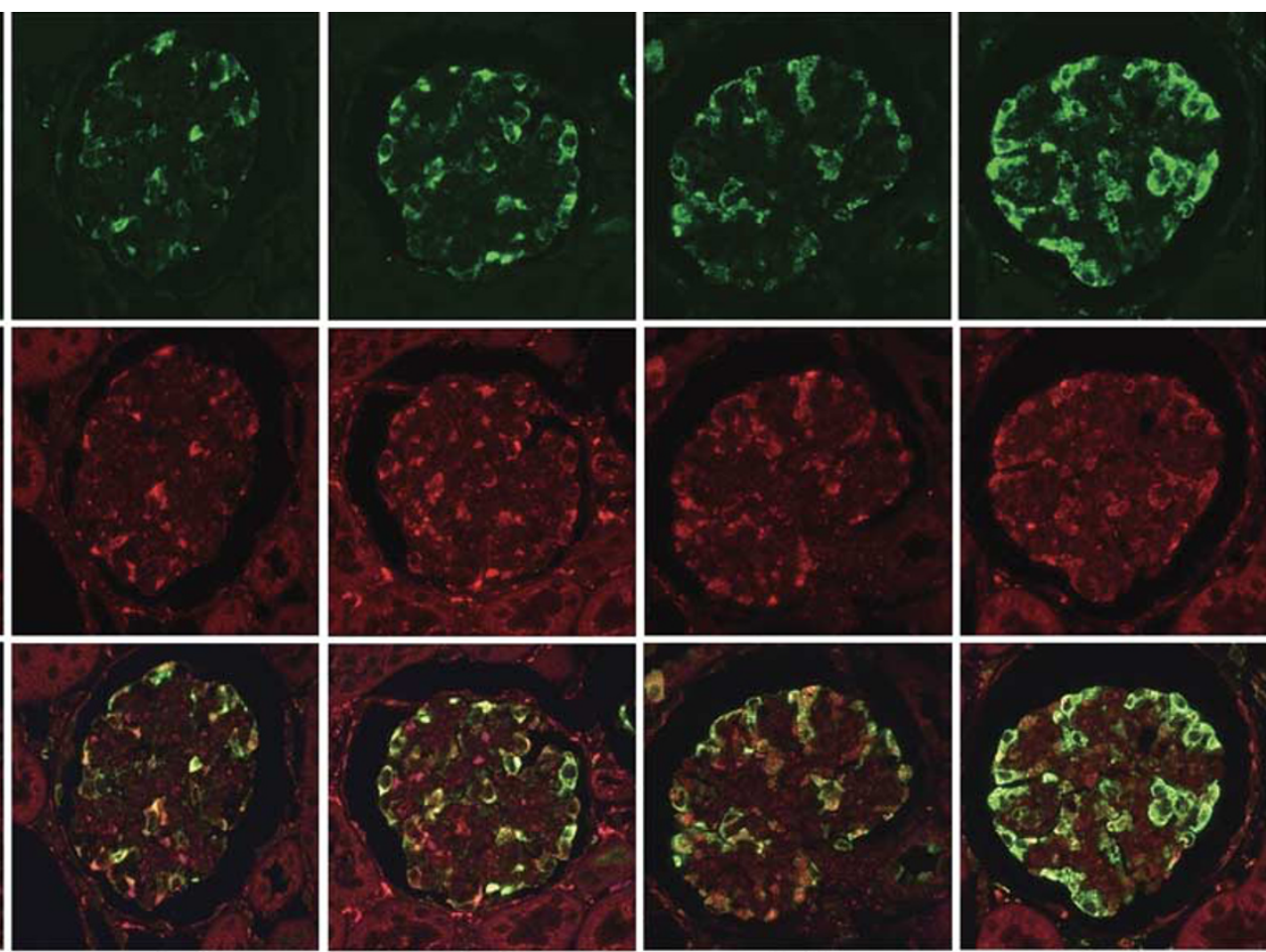

day 1

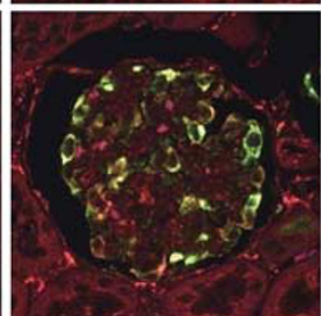

day 2

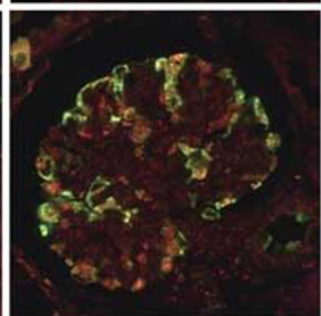

day 3

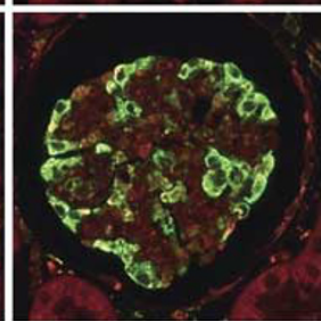

day 5

Figure 4 mTORC1 activation and ER stress during PAN nephrosis. Dual-immunofluorescence and confocal microscopy for phospho-S6 ribosomal protein (pS6RP) (green) and the ER stress marker, GRP78 (red), in the kidney sections from PAN nephrosis samples. Original magnification, $\times 400$.

increased, and this clearly overlapped with GRP78 expression in the podocyte cytoplasm (Figure 4). The expression of both p-S6RP and GRP78 in the podocytes continued to increase on day 2 and persisted until day 5 (Figure 4). To examine whether ER stress indeed induces UPR activation and to determine how both UPR and mTORC1 activation progress during proteinuria, western blot with S6RP for mTORC1 and eIF $2 \alpha$ for UPR in addition to GRP78 was conducted. It is still unclear whether real signal activation inside cells should be estimated by the ratio of the phosphorylated form of the protein to its substrate (total form) because the signal activation itself also induces the production of its substrate and accelerates its protein turnover. Nevertheless, when the ratios of the phosphorylated form to the total form were compared, the mTORC1 activation commenced from day 1 and further increased on day 2 (Figure 5). On and after day 3, this tended to decrease to baseline level. The UPR activation also tended to commence from day 1 and further increased until day 3 , and then decreased to base line level on day 5 (Figure 5). When estimated by the ratio of the phosphorylated form of both S6RP and eIF $2 \alpha$ to $\beta$-actin, their activation clearly continued even on day 5 (data not shown). To further confirm the predominant mTORC1 activation in the glomeruli from PAN-induced nephrosis, western blot using antibodies against phosphorylated-p70S6K (Thr389) and 4EBP1 (Ser65) was also examined. When compared with $\beta$-actin, activation of p70S6K (Thr389) behaved like S6RP, which already commenced to be activated from day 1 and continued until day 5 (Figure 6). Activation of 4EBP1 (Ser65) also occurred from day 1; however, its intensity tended to decrease between day 2 and day 3, and again increased on day 5 (Figure 6). In these samples, the activation of AMPK $\alpha$, a sensor of the AMP/ATP ratio within cells, was also evaluated. Interestingly, this was rather attenuated on day 1, followed by strong activation from day 2, and continued until day 5 (Figure 6). Phosphorylation of Akt at Ser473 is activated by mTORC2, a rapamycin-insensitive complex with rictor and Sin1. In the same glomerular samples, there was not apparent activation of Akt at Ser473, suggesting the predominant activation of mTORC1 associated with UPR in the series of the glomeruli from PAN-induced nephrosis (Figure 6).

\section{mTORC1 Activation Precedes UPR in Cultured Podocytes}

Despite vigorous experiments employing glomerular samples with western blots, we were unable to distinguish the conclusive time lag of the activation between mTORC1 and UPR pathways. This was most likely due to the time-consuming method for glomerular isolation that at least required $6 \mathrm{~h}$, thereby influencing the state of very sensitive signaling pathway in the samples. Therefore, we next employed immortalized podocytes that were treated with PAN in a timedependent manner. As shown in Figure 7, the 3-h treatment 

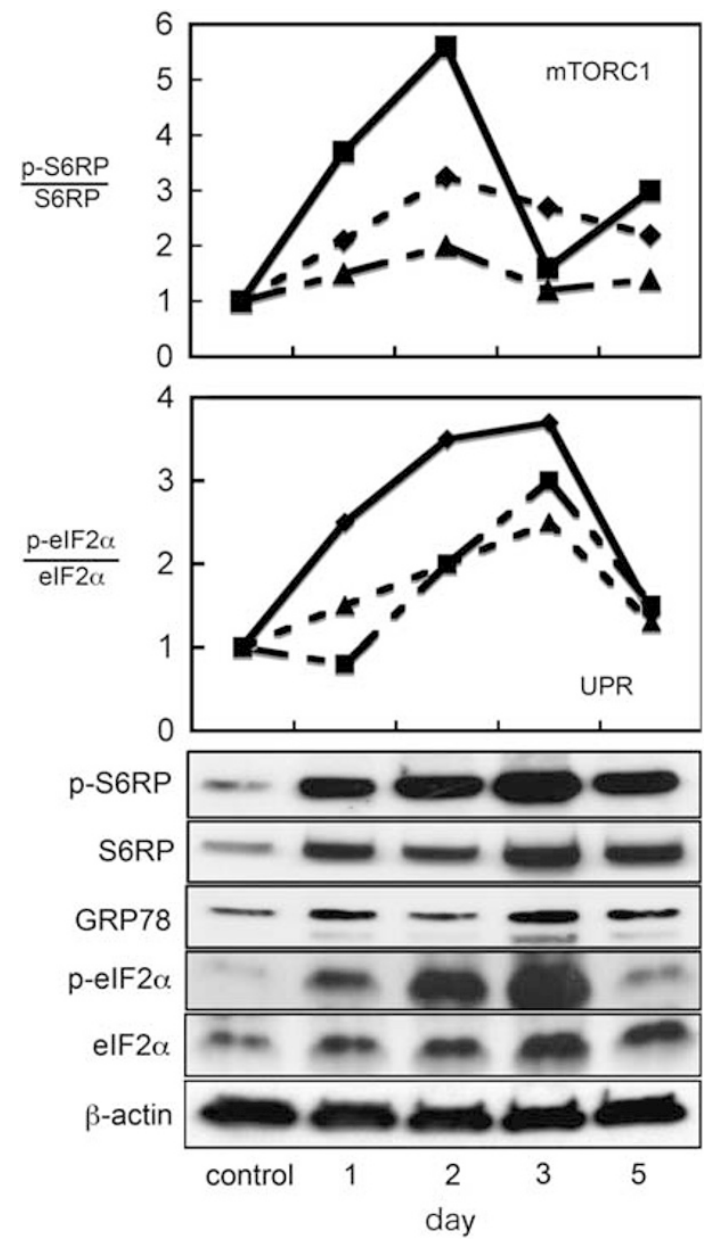

Figure 5 Co-activation of mTORC1 and UPR in PAN glomeruli. Western blot analysis using the indicated antibodies and lysates from isolated glomerular samples from PAN nephrosis and control rats. Semi-quantitative analysis of the protein density from the western blot $(n=3)$ was calculated by the ratio of the phosphorylated form to the total form.

with PAN activated the mTORC1 pathway, whereas UPR activation was not observed. On and after $24 \mathrm{~h}$, mTORC1 activation tended to revert to a baseline level, whereas UPR was eventually activated. Expression of GRP78 clearly behaved like UPR activation through $48 \mathrm{~h}$. The energy charge, which is defined as $((\mathrm{ATP})+1 / 2(\mathrm{ADP})) /((\mathrm{ATP})+$ $(\mathrm{ADP})+(\mathrm{AMP}))$ describes the cellular balance between the energy-consuming state and the energy-producing state. ${ }^{24}$ The energy charge implies that AMP drives the cellular energy balance toward the energy-producing adaptation. ${ }^{25}$ As depicted in Figures 8a and b, the ATP levels markedly decreased after $24 \mathrm{~h}$ with a concurrent increase in the AMP levels, whereas the ADP levels showed no significant changes, which induced a marked decrease in the energy charge between 3 and $24 \mathrm{~h}$ of incubation. Thus, the cells appeared to be shifted to an energy-deficient state by the prolonged incubation. Indeed, although there was no significant activation of AMPK $\alpha$ at the 3-h time point, its clear activation

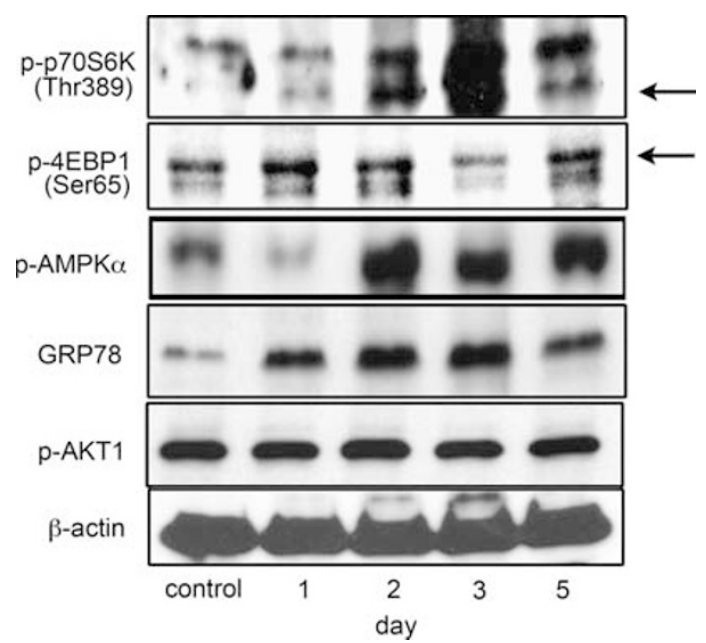

Figure 6 Predominant activation of mTORC1 in PAN glomeruli. Specific downstream molecules of mTORC1, p-70S6K (Thr389) and 4EBP1 (Ser65), were clearly activated from day 1 , whereas phosphorylation of AKT1 (Ser473), the site regulated by mTORC2 activity, was not enhanced. Activation of AMPK $\alpha$, a sensor of the AMP/ATP ratio within cells, commenced on day 2 , not on day 1 .

was observed at $24 \mathrm{~h}$ and continued until $48 \mathrm{~h}$ (Figures $8 \mathrm{c}$ and d). Interestingly, pre-treatment with everolimus clearly abrogated the activation of eIF $2 \alpha$ and AMPK $\alpha$ as well as S6RP and protected GRP78 upregulation, suggesting that mTORC1 activation was an actual trigger of UPR in cultured podocytes treated with PAN (Figure 8e).

mTOR Inhibitor Interferes Proteinuria in PAN Nephrosis Finally, we sought to verify whether mTORC1 activation could indeed act as an upstream to induce UPR in the MCD model. Daily treatment with everolimus at a $4 \mathrm{mg} / \mathrm{kg} / \mathrm{day}$ dose was administered 1 day before the PAN injection and was continued for 6 days. The serum everolimus concentration at $2 \mathrm{~h}$ after gavage on day 5 was $3.33 \pm 1.63 \mathrm{ng} / \mathrm{ml}$ (mean \pm s.d., $n=3$ ). No everolimus signal was detected in the sera from vehicle-treated rats. The pre-treatment with everolimus clearly protected against proteinuria (Figure 9). This protective effect of everolimus appeared to be due to the preservation of nephrin expression, which was determined using immunofluorescence and confocal microscopy and western blot (Figures 10a and b). Reduction of mTORC1 and UPR activation in the glomeruli of everolimus-treated rats compared with vehicle-treated one was apparently demonstrated by semi-quantitative analysis with western blots and immunofluorescence microscopy (Figures 10c-e).

\section{DISUCUSSION}

Our study is the first to suggest that mTORC1 activation is the upstream of ER stress in the MCD model, which secondarily induces the UPR activation that underlies the pathogenesis of proteinuria by arresting protein translation of podocyte-producing molecules, including nephrin. 


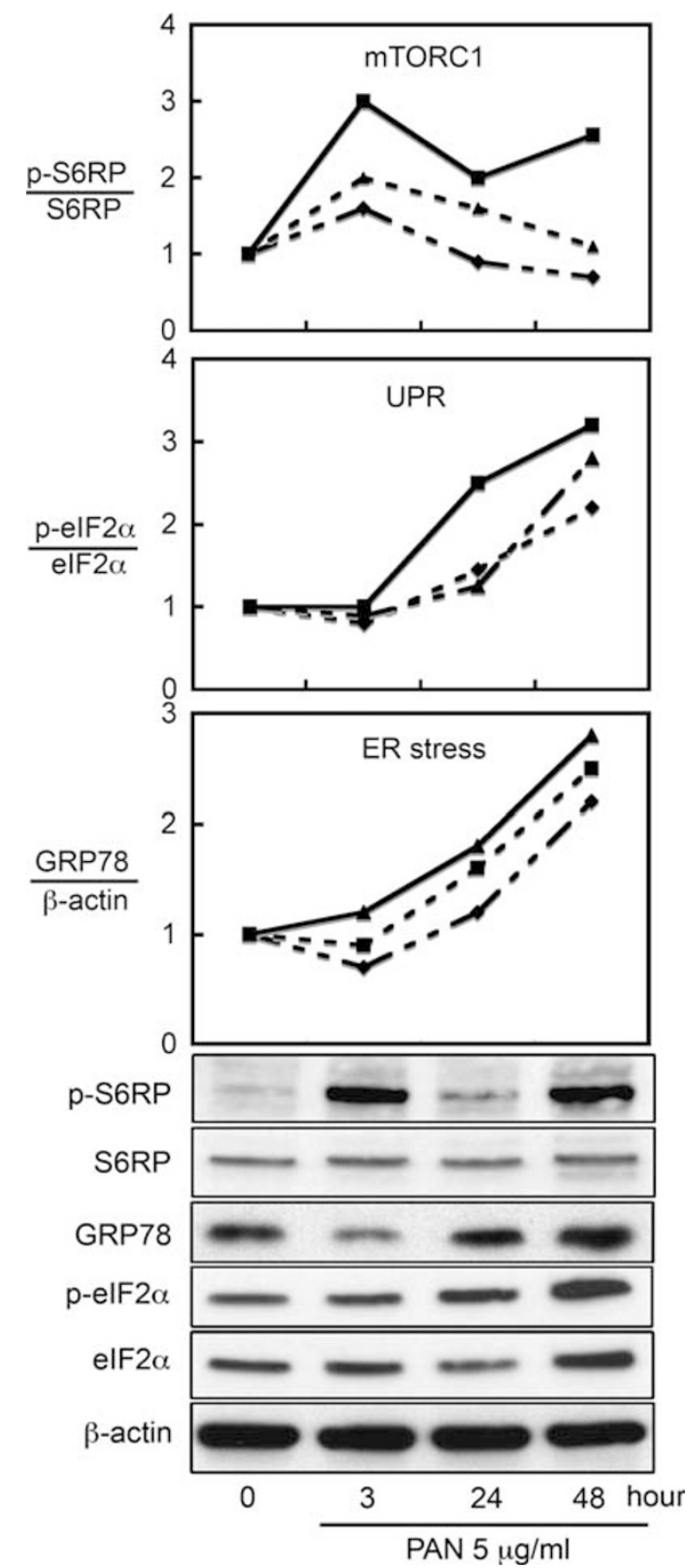

Figure 7 mTORC1 activation before UPR in PAN-treated cultured podocytes. Western blot using the indicated antibodies and lysates from the samples from cultured mouse podocytes treated with PAN $(5 \mu \mathrm{g} / \mathrm{ml})$. Semi-quantitative analysis of the protein density from the western blot indicated. The ratio of the phosphorylated form to the total form was plotted. GRP78 expression was corrected by $\beta$-actin. Three experiments were examined for each time point.

In mammalian cells, the UPR signaling pathways are initiated by three ER stress sensors: inositol-requiring $1 \alpha$, double-stranded RNA-dependent protein kinase-like ER kinase and activating transcription factor 6 , in which doublestranded RNA-dependent protein kinase-like ER kinase has protein kinase activity and stimulates eIF $2 \alpha$ phosphorylation that inhibits the assembly of the $80 \mathrm{~S}$ ribosome and, conse- quently interferes the synthesis of proteins. ${ }^{26,27}$ With regard to the involvement of ER stress and UPR activation in the pathogenesis of glomerulopathy, Cybulsky et al ${ }^{28}$ demonstrated that phosphorylation of eIF2 $\alpha$ and the expression of GRP78 were enhanced significantly in glomeruli of proteinuric rats with passive Heymann nephritis, a model of human membranous nephropathy. Inagi et $a l^{29}$ reported a pathogenic role of accumulation of misfolded protein in the ER of podocytes in the pathogenesis of proteinuria by using a transgenic rat overexpressing mature and mutant megsin, a member of the serine protease inhibitor family. More recently, we revealed strong induction of GRP78 in the glomeruli at the heavy proteinuric state in rat MCD induced by PAN. ${ }^{9}$ These results suggested the definite implication of ER stress in the pathogenesis of proteinuria; however, whether this ER stress was a cause or a concurrence of podocyte dysfunction and how and when UPR activation occurred in the diseased glomerular podocytes remained elusive. This study clearly showed that UPR demonstrated by eIF $\alpha$ was already activated and associated with the upregulation of GRP78 on day 1 glomeruli in PAN nephrosis (Figure 5). Another new crucial event at this time point was the alteration of nephrin localization. Despite the preservation of so-called plasma membrane form nephrin $(\sim 200 \mathrm{kDa})$ that was shown by western blot (Figure 3), there was clear nephrin mislocalization that was observed in the podocyte cytoplasm, different from its proper localization at the plasma membrane, already in day 1 glomeruli as well as day 2 glomeruli (Figure 2b). Moreover, we also identified that so-called ER form nephrin $(\sim 175 \mathrm{kDa})$ was already reduced on days 1 and 2, whereas nephrin mRNA was conversely upregulated (Figures $3 c$ and $d$ ). This strongly suggests that UPR activation in podocytes induced by ER stress was a cause of pathological proteinuria, which arrested the protein production of new nephrin at the post-translational level and influenced nephrin localization, thereby disrupting a permselective barrier function of the slit diaphragm. How UPR activation was directly linked to mislocalization of nephrin in the glomerular podocyte is still obscure; however, a presence of lower molecular weight nephrin compared with $\sim 175-\mathrm{kDa}$ ER nephrin indicates that ER stress might interfere the post-translational modification system of nephrin such as glycosylation in the ER.

Both in animals in vivo and cells in vitro, protein synthesis is regulated through a signaling pathway involving mTORC1 $1{ }^{30}$ In this regard, activation of mTORC1 stimulates both the initiation and elongation phases of mRNA translation. In addition, mTORC1 activation is associated with increased cell-cycle progression, selective enhancements in gene transcription, and increased ribosome biogenesis, results in cell size enlargement and cell proliferation. ${ }^{31}$ This means that signaling through mTORC1 drives many energyconsuming processes. Since a previous paper showed the protective effect of mTOR inhibitor in PAN nephrosis, ${ }^{16}$ we 

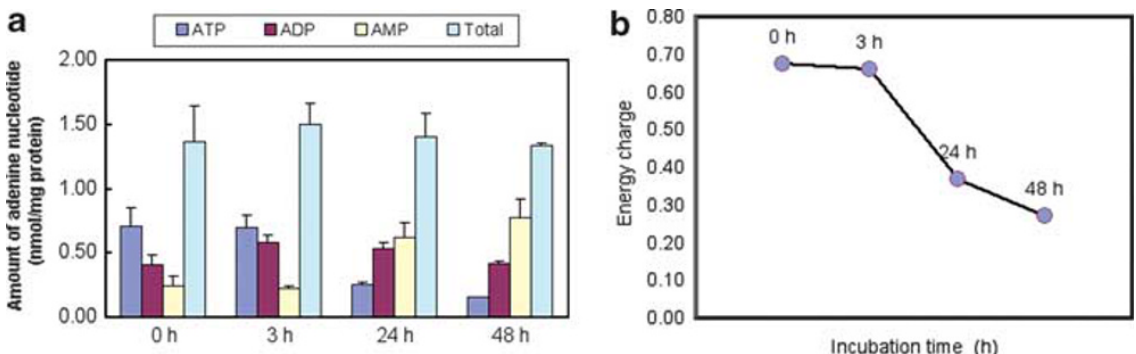

C
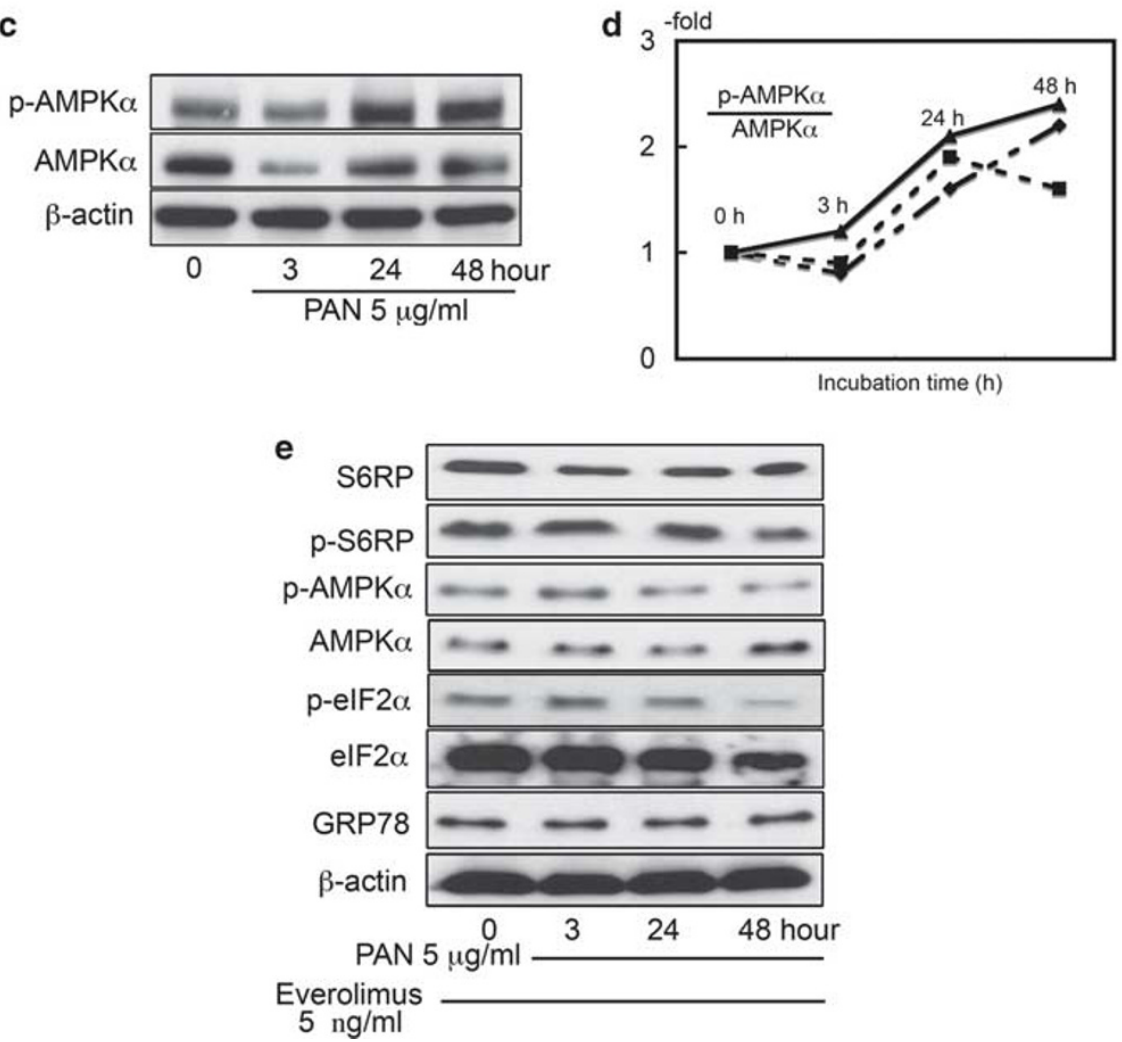

Figure 8 The level of energy in PAN-treated cultured podocytes. (a) Measurement of adenine nucleotides in the same samples ( $n=3$ per group) from Figure 7. (b) The energy charge calculated as described in the text: ((ATP) $+1 / 2($ ADP))/((ATP) + (ADP) + (AMP)). (c, d) The state of energy consumption was further evaluated by AMPK $\alpha$ activation shown by western blot, followed by semi-quantitative analysis. (e) Pre-treatment with everolimus abrogated AMPK $\alpha$ activation in association with the protection against ER stress in PAN-treated podocytes.

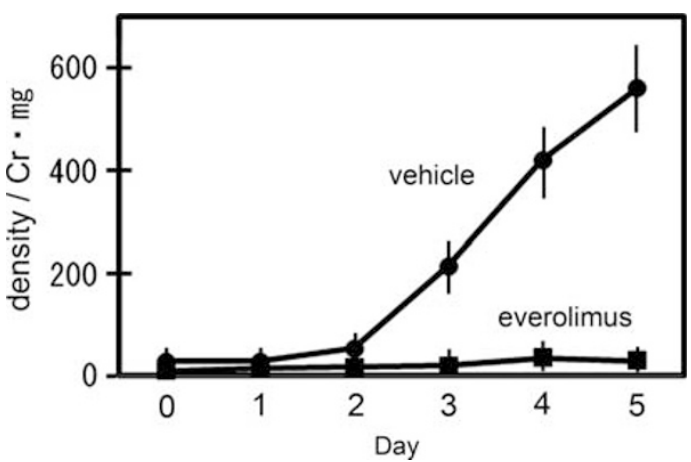

Figure 9 The effect of the mTOR inhibitor everolimus on urinary protein in PAN nephrosis. Semi-quantitative analysis of the urinary protein determined using Coomassie blue staining/ $\mathrm{Cr} \mathrm{mg}$ ratio ( $n=3$ per group). hypothesized that an energy-consuming process may precede UPR in podocytes with MCD. Our data revealed the simultaneous activation of both mTORC1 and UPR pathways in podocytes on day 1 glomeruli before proteinuria. However, in vitro study using cultured podocytes treated with PAN further determined that mTORC1 activation clearly preceded UPR activation. Moreover, in vivo study using everolimus apparently revealed that the inhibition of mTORC1 activation interfered the pathological proteinuria in PAN nephrosis, which was associated with the reduction of both mTORC1 and UPR activation. Thus, it is most likely that mTORC1 activation in podocytes behaved as the upstream of UPR. The data from western blot with AMPK $\alpha$ activation and the measurement of intracellular adenine 


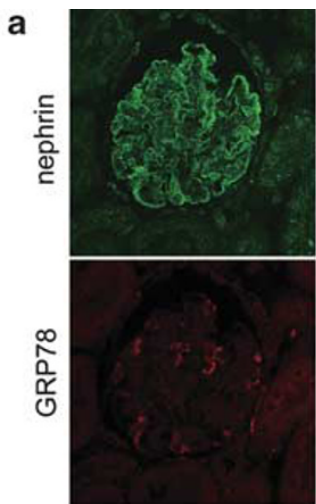

control
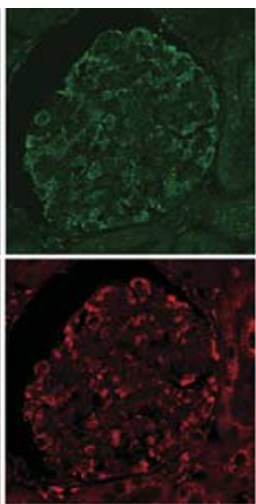

vehicle

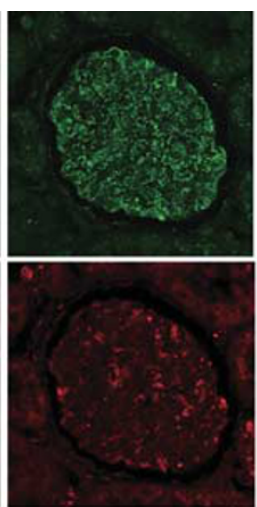

everolimus b

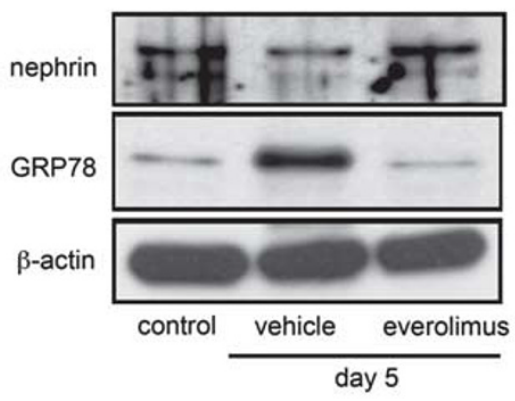

day 5
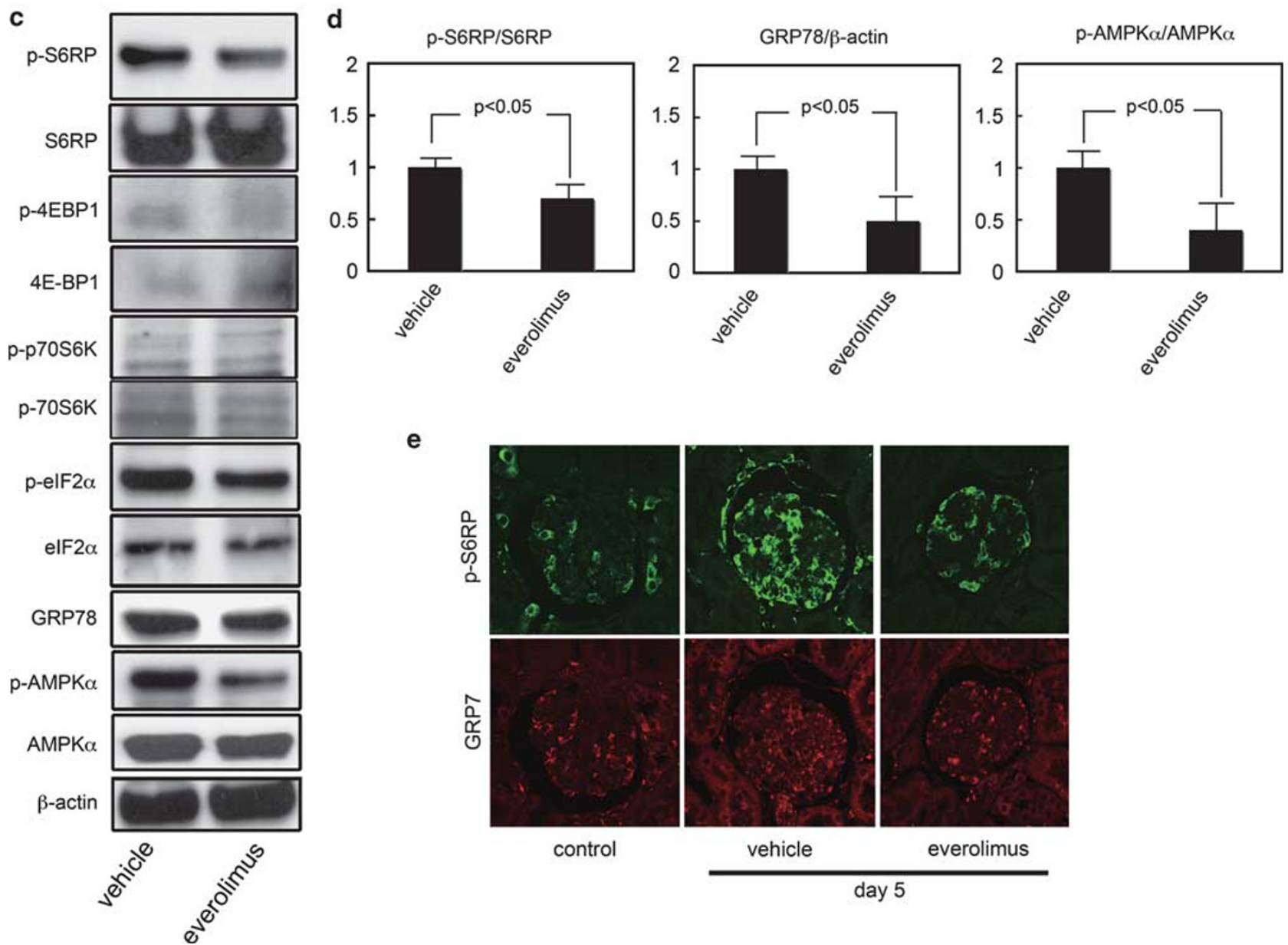

e
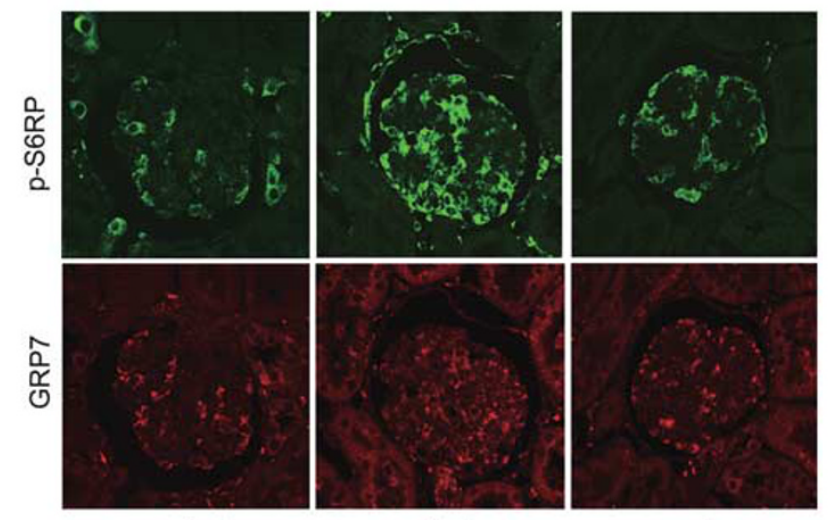

control

vehicle

everolimus

day 5

Figure 10 The effect of the mTOR inhibitor everolimus on the activation of mTORC1, UPR and nephrin. (a) Dual-immunofluorescence and confocal microscopy with nephrin and GRP78. (b) PAN administration induced a drastic reduction of both form of nephrin, which was clearly protected by pretreatment with everolimus. (c) Western blotting used for the antibodies indicated and the glomerular samples from vehicle-treated and everolimus-treated PAN rats. (d) Semi-quantitative analysis with (c), mean \pm s.e.m., $n=3$ rats. (e) Dual-immunofluorescence and confocal microscopy with phosphorylated S6RP and nephrin and GRP78. Original magnification, $\times 400(\mathbf{a}, \mathbf{e})$.

nucleotides suggested that mTORC1 activation really consumed energy in podocytes, which in turn activated UPR for the podocyte survival.
Previous in vitro study showed that prolonged rapamycin treatment reduced mTOR activation and led to the decreased expression of the slit diaphragm components of cultured 
podocytes. ${ }^{32}$ Torras et al also reported that rapamycin provoked significant increase in proteinrua in PAN. ${ }^{33}$ These contradictory results against our present data might be evoked by the difference of the start point to treat cells and animals with mTOR inhibitor although both previous papers did not describe its start point in detail. Indeed, when everolimus was started just after or 1 day after PAN injection, we did not found any its protective effect in the development of proteinuria (data not shown). Moreover, mTOR inhibitors that are used in kidney and heart transplant patients have been known to cause proteinuria. ${ }^{34,35}$ However, in such cases, whether the inhibition of the mTOR pathway is an initial trigger of podocyte damage is unclear because transplanted patients had already been treated with calcineurin inhibitors and glucocorticoids before being switched to the mTOR inhibitors. On the other hand, the simultaneous activation of mTORC1 and UPR in our in vivo study also appears to be contradictory. However, Ozcan et $a l^{36}$ recently reported that the loss of the tuberous sclerosis complex genes, which suppress mTORC1, led to the constitutive activation of the mTORC1 pathway and also caused ER stress, which in turn activated the UPR. This study and the present data suggested that the simultaneous activation of mTORC1 and the UPR in cells could indeed exist. As all cells must possess only a limited adaptive capacity to energy stress and metabolic dysregulation, the activities of these opposite survival pathways may be often coordinately regulated. Therefore, it is not surprising to uncover a link between ER-stress and the mTORC1 pathway, which is a critical component of the pathologies of many glomerular disorders that are associated with proteinuria. Our data also suggest that the energy regulatory system in podocytes may be a target of the conventional glucocorticoids and immunosuppressive agents that are used to treat MCD.

Supplementary Information accompanies the paper on the Laboratory Investigation website (http://www.laboratoryinvestigation.org)

\section{ACKNOWLEDGEMENT}

We thank Y Kimura for her excellent technical assistance.

\section{DISCLOSURE/CONFLICT OF INTEREST}

The authors declare no conflict of interest.

1. Ma XM, Blenis J. Molecular mechanisms of mTOR-mediated translational control. Nat Rev Mol Cell Biol 2009;10:307-318.

2. Schröder M. Endoplasmic reticulum response. Cell Mol Life Sci 2008;65:862-894.

3. Anelli T, Sitia R. Protein quality control in the early secretory pathway. EMBO J 2008:27:315-327.

4. Hegde RS, Ploegh HL. Quality and quantity control at the endoplasmic reticulum. Curr Opin Cell Biol 2010;22:437-446.

5. Ni M, Lee AS. ER chaperones in mammalian development and human diseases. FEBS Lett 2007;581:3641-3651.

6. Kaufman RJ. Orchestrating the unfolded protein response in health and disease. J Clin Invest 2002;110:1389-1398.

7. Cybulsky AV. Endoplasmic reticulum stress in proteinuric kidney disease. Kidney Int 2010;77:187-193.
8. Dickhout JG, Krepinsky JC. Endoplasmic reticulum stress and renal disease. Antioxid Redox Signal 2009;11:2341-2352.

9. Nakajo A, Khoshnoodi J, Takenaka $\mathrm{H}$, et al. Mizoribine corrects defective nephrin biogenesis by restoring intracellular energy balance. J Am Soc Nephrol 2007;18:2554-2564.

10. Mathieson PW. Minimal change nephropathy and focal segmental glomerulosclerosis. Semin Immunopathol 2007;29:415-426.

11. Schönenberger $E$, Ehrich $J \mathrm{H}$, Haller $\mathrm{H}$, et al. The podocyte as a direct target of immunosuppressive agents. Nephrol Dial Transplant 2011;26:18-24.

12. Kestilä $M$, Lenkkeri $U$, Männikkö $M$, et al. Positionally cloned gene for a novel glomerular protein-nephrin-is mutated in congenital nephrotic syndrome. Mol Cell 1998;1:575-582.

13. Lenkkeri U, Männikkö $M, M c C r e a d y ~ P$, et al. Structure of the gene for congenital nephrotic syndrome of the finnish type (NPHS1) and characterization of mutations. Am J Hum Genet 1999;64: 51-61.

14. Yan K, Khoshnoodi J, Ruotsalainen V, et al. N-linked glycosylation is critical for the plasma membrane localization of nephrin. J Am Soc Nephrol 2002;13:1385-1389.

15. Fujii $\mathrm{Y}$, Khoshnoodi J, Takenaka $\mathrm{H}$, et al. The effect of dexamethasone on defective nephrin transport caused by ER stress: A potential mechanism for the therapeutic action of glucocorticoids in the acquired glomerular diseases. Kidney Int 2006;69:1350-1359.

16. Daniel C, Ziswiler R, Frey B, et al. Proinflammatory effects in experimental mesangial proliferative glomerulonephritis of the immunosuppressive agent SDZ RAD, a rapamycin derivative. Exp Nephrol 2000;8:52-62.

17. Yang Q, Guan KL. Expanding mTOR signaling. Cell Res 2007;17: 666-681.

18. Wang $\mathrm{X}$, Campbell $\mathrm{LE}$, Miller $\mathrm{CM}$, et al. Amino acid availability regulates p70 S6 kinase and multiple translation factors. Biochem J 1998;334:261-267.

19. Fingar DC, Richardson CJ, Tee AR, et al. mTOR controls cell cycle progression through its cell growth effectors S6K1 and 4E-BP1/ eukaryotic translation initiation factor 4E. Mol Cell Biol 2004;24: 200-216.

20. Hay N, Sonenberg N. Upstream and downstream of mTOR. Genes Dev 2004;18:1926-1945.

21. Shimizu M, Khoshnoodi J, Akimoto Y, et al. Expression of galectin-1, as a new component of slit diaphragm, is altered in minimal change nephritic syndrome. Lab Invest 2009;89:178-195.

22. Sekine $Y$, Nishibori $Y$, Akimoto $Y$, et al. Amino acid transporter LAT3 is required for podocyte development and function. J Am Soc Nephrol 2009;20:1586-1596.

23. Kurayama $\mathrm{R}$, Ito $\mathrm{N}$, Nishibori $\mathrm{Y}$, et al. Role of amino acid transporter LAT2 in the activation of mTORC1 pathway and the pathogenesis of crescentic glomerulonephritis. Lab Invest 2011;91: 992-1006.

24. Ramaiah A, Hathaway JA, Atkinson DE. Adenylate as a metabolic regulator. Effect on yeast phosphofructokinase kinetics. J Biol Chem 1964;239:3619-3622.

25. Hardie DG, Hawley SA. AMP-activated protein kinase: the energy charge hypothesis revisited. BioEssays 2001;23:1112-1119.

26. Mandl J, Mészáros T, Bánhegyi G, et al. Endoplasmic reticulum: nutrient sensor in physiology and pathology. Trends Endocrinol Metab 2009;20:194-201.

27. Malhotra JD, Kaufman RJ. The endoplasmic reticulum and the unfolded protein response. Semin Cell Dev Biol 2007;18:716-731.

28. Cybulsky AV, Takano T, Papillon J, et al. Role of the endoplasmic reticulum unfolded protein response in glomerular epithelial cell injury. J Biol Chem 2005;280:24396-24403.

29. Inagi $R$, Nangaku $M$, Usuda $N$, et al. Novel serpinopathy in rat kidney and pancreas induced by overexpression of megsin. J Am Soc Nephrol 2005;16:1339-1349.

30. Abraham RT. TOR signaling: an odyssey from cellular stress to the cell growth machinery. Curr Biol 2005;15:R139-R141.

31. Fingar DC, Blenis J. Target of rapamycin (TOR): an integrator of nutrient and growth factor signals and coordinator of cell growth and cell cycle progression. Oncogene 2004;23:3151-3171.

32. Vollenbröker $\mathrm{B}$, George $\mathrm{B}$, Wolfgart $\mathrm{M}$, et al. mTOR regulates expression of slit diaphragm proteins and cytoskeleton structure in podocytes. Am J Physiol Renal Physiol 2009;296:F418-F422. 
33. Torras J, Herrero-Fresneda I, Gulias $\mathrm{O}$, et al. Rapamycin has dual opposing effects on proteinuric experimental nephropathies: is it a matter of podocyte damage? Nephrol Dial Transplant 2009;24: 3632-3640.

34. Sánchez-Fructuoso $\mathrm{Al}$, Ruiz JC, Pérez-Flores $\mathrm{I}$, et al. Comparative analysis of adverse events requiring suspension of mTOR inhibitors: everolimus vs sirolimus. Transplant Proc 2010;42: 3050-3052.
35. Schena FP, Pascoe MD, Alberu J, et al. Sirolimus CONVERT Trial Study Group. Conversion from calcineurin inhibitors to sirolimus maintenance therapy in renal allograft recipients: 24-month efficacy and safety results from the CONVERT trial. Transplantation 2009;87:233-242.

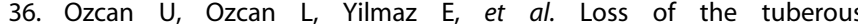
sclerosis complex tumor suppressors triggers the unfolded protein response to regulate insulin signaling and apoptosis. Mol Cell 2008;29:541-551. 